\title{
IMPACTO DEL MEDIO AMBIENTE EN LA CULTURA ESTUDIANTIL DE LA UNIVERSIDAD TÉCNICAS ESTATAL DE QUEVEDO.
}

\section{IMPACT OF THE ENVIRONMENT IN THE STUDENT CULTURE OF THE STATE TECHNICAL UNIVERSITY OF QUEVEDO.}

Jhon Alejandro Boza Valle, PhD.

Doctor en Ciencias Económicas (Cuba).

Docente Titular de la Facultad de Ciencias Empresariales de la Universidad Técnica Estatal de Quevedo, Ecuador. jboza@uteq.edu.ec Henry Caiza Villareal, Ec.

Economista (Ecuador). henrypacrilex_k@hotmail.com

Emma Yolanda Mendoza Vargas, Mgs.

Magíster en Dirección de Empresas con énfasis en Gerencia estratégica (Ecuador). Docente la Unidad de Estudios a Distancia de la Universidad Técnica Estatal de Quevedo, Ecuador. emendoza@uteq.edu.ec Manuel Francisco Morales Haz, Mgs. Magíster en enseñanza de inglés como idioma extranjero (Ecuador). Docente de Facultad de Ciencias Empresariales de la Universidad Técnica Estatal de Quevedo, Ecuador. mfmorales@uteq.edu.ec

\section{ARTÍCULO DE INVESTIGACIÓN}

Recibido: 26 de enero de 2018

Aceptado: 23 de marzo de 2018

\section{RESUMEN}

La cultura ambiental es la manera como los seres humanos interactúan con el medio ambiente. El presente ensayo pretende determinar la cultura ambiental en los estudiantes de la Universidad Técnica Estatal de Quevedo enfocado en el estudio de los valores éticos y morales del ser humano hacia el cuidado del medio ambiente con el 
fin de establecer creencias, actitudes y comportamientos ecológicos para preservar un ambiente saludable y sustentable. También se pretende concientizar al hombre a cuidar el entorno ambiental con responsabilidad y respeto. Estableciendo una educación ambiental correcta con características propias de la colectividad. La investigación utiliza el método analítico, en revisión de las aptitudes ambientales negativas del estudiante en el centro universitario y el método descriptivo y exploratorio para describir los posibles fallos que ocurren en contra de la naturaleza. Este trabajo contribuirá a mejorar la calidad de vida mejorando, el bienestar y el desarrollo institucional. Se pretende proyectar una imagen diferente a la colectividad universitaria de actitud positiva con eficiencia y liderazgo.

Palabras clave: cultura ambiental, educación ambiental, ecosistema, ética y moral.

\section{ABSTRACT}

Environmental culture is the way humans interact with the environment, this essay aims to determine the environmental culture in the students of Quevedo State Technical University focused on the study of ethical and moral values of the human being towards care Of the environmental ecosystem, which establishes beliefs, attitudes and ecological behaviors to preserve a healthy and sustainable environment, to make people aware of the environment with responsibility and respect, it is necessary to establish a correct environmental education with characteristics of the community, research uses the analytical method in reviewing the negative environmental skills of the student in the university center in which the descriptive and exploratory method is triggered describing possible failures against nature, improving the quality of life, well-being and development institution $\mathrm{Al}$, is to project a different image to the university community of positive attitude with efficiency and leadership.

Keywords: Environmental culture, environmental education, ecosystem, ethics and morals.

\section{INTRODUCCIÓN}

La presente investigación se basa en la escasa cultura ambiental en los estudiantes de la Universidad Técnica Estatal de Quevedo y su impacto en el medio ambiente, el cual 
es de suma importancia para todos los actores sociales de la universidad, que con responsabilidad deben cuidar actitudes para preservar el medio ambiente.

Mediante el análisis de la relación entre el medio ambiente y cultura en general, el entorno en el cual opera una organización, que incluye el aire, el agua, el suelo, los recursos naturales, la flora, la fauna, los seres humanos, y su interrelación. En este contexto, el medio ambiente se extiende desde el interior de una organización hasta el sistema global.

\section{REVISIÓN TEÓRICA}

El medio ambiente entonces se refiere a todo lo que rodea a los seres vivos, está conformado por elementos biofísicos (suelo, agua, clima, atmósfera, plantas, animales y microorganismos), y las relaciones con la cultura se manifiestan además de la ideología y economía. La relación que se establece entre estos elementos es lo que, desde una visión integral, conceptualiza el medio ambiente como un sistema (Arango, 2015), en la actualidad el concepto de medio ambiente está ligado al de desarrollo; esta relación nos permite entender los problemas ambientales y su vínculo con el desarrollo sustentable, el cual debe garantizar una adecuada calidad de vida para las generaciones presente y futura.

Debido a esto es necesario cambiar la concepción y el acercamiento a la realidad que tienen los individuos. Se enfoca en la educación ambiental de acuerdo con las características propias de la comunidad y se hace importante desarrollar estudios en los cuales se determinen estas variables o dimensiones, si se quiere avanzar en la consolidación de la cultura ambiental favorable con el medio ambiente y, por ende, progresar en la búsqueda de la sostenibilidad.

La relación entre las tendencias culturales como sistemas de creencias, valores compartidos, actitudes podría dar cuenta de los comportamientos y estrategias que los individuos mantienen en la interacción con el medio ambiente". Nótese que la cultura ambiental tiene relación directa con el individuo, donde sus principios morales reflejan el desempeño bueno o malo con el medio ambiente, también dependerá mucho de su formación cultural aquel desempeño. Por lo tanto, dichas variables, en su más amplio 
sentido, tienen un peso determinante en el desarrollo de una cultura ambiental sostenible.

Por otra parte (Miranda, 2013) destaca: "El compromiso con valores, creencias y actitudes más próximos a una relación armónica con el medio ambiente podría convertirse en un poderoso predictor del cambio de los contextos en los comportamientos. Por ello, el análisis de la interacción entre las dimensiones mencionadas mostraría de una forma más amplia los aspectos más relevantes implicados en el desarrollo de una cultura ambiental".

Sin embargo, la educación ambiental es muy amplia, pero se pueden distinguir dos grandes corrientes que lo caracterizan. La primera contempla la enseñanza de las ciencias naturales y se define como la educación acerca del ambiente; la segunda, que es en la que puedes participar de manera activa en la construcción y participación de proyectos y actividades fuera del aula, denominada educación en el ambiente, y que sirve para proponer soluciones en beneficio del ambiente y del desarrollo del ser humano en equilibrio con este (Aguilar, 2011). A través del estudio, se puede entender que la cultura y la educación ambiental son herramientas primordiales para el cambio o mejoramiento de actitudes del individuo a favor del ecosistema, se incluye en proyectos académicos desde niveles básicos hasta universitarios con el apoyo de las autoridades.

Según (Rengifo \& Quitiatez) manifiesta que los procesos educativos que incluyen la enseñanza de la educación ambiental pretenden tomar conciencia del individuo y la sociedad de igual forma la capacitación conforman un proceso que permite que los seres humanos y las sociedades desarrollen plenamente el conocimiento del entorno, interpretarlos, explicarlos y vivir sus circunstancias.

Del mismo modo educación ambiental promueve el desarrollo y aumenta las posibilidades de la población para emprender su desenvolvimiento. Si bien la educación básica sirve de fundamento para la educación en materia de medio ambiente y desarrollo, esta última debe incorporarse como parte fundamental del aprendizaje. La educación ambiental es un eje dinamizador para modificar las actitudes de las personas de manera que éstas sean capaces de evaluar los problemas de desarrollo sostenible o sustentable y abordarlos de manera adecuada. 
Declara también que la educación ambiental es un proceso que reconoce valores y aclarar conceptos centrados en fomentar las actitudes, destrezas, habilidades y aptitudes necesarias para comprender y apreciar las interrelaciones entre el ser humano, su cultura y la interrelación con la naturaleza.

La educación es fundamental para adquirir conciencia, valores, técnicas y comportamientos ecológicos y éticos en consonancia con el desarrollo sostenible y que favorezcan la participación comunitaria efectiva en decisiones. Así lo afirma la conferencia de toma de decisiones, Naciones Unidas (1992, p.20). Por lo consiguiente, educación ambiental toma en cuenta el entorno natural y artificial en su totalidad: ecológico, social, cultural y estético; debería ser un proceso continuo y permanente en la Universidad y fuera de ella; tener un enfoque interdisciplinario, hacer hincapié en una participación activa en la prevención y solución de los problemas ambientales desde un punto de vista general, teniendo en cuenta las diferencias regionales; debería concentrarse en cuestiones ambientales actuales y futuras; debería considerar desarrollo y crecimiento en una perspectiva ambiental; la educación en fomentar el valor y la necesidad de la cooperación local, nacional e internacional en la resolución de los problemas ambientales.

En este sentido, se considera que la educación ambiental debe ser un eje transversal común al currículum de la educación superior, para que los estudiantes obtengan conocimientos, se sensibilicen con respecto a su entorno y adquieran valores que promueva un comportamiento favorable hacia el ambiente.

Por otra parte, el impacto ambiental comprende los cambios o modificaciones que afectan al ambiente, ocasionados por determinadas obras, acciones o servicios del hombre, con implicaciones ambientales o eventos ocasionales de la naturaleza, con efectos en la calidad ambiental y de vida, y en el aprovechamiento de los recursos naturales.

En paralelo la evolución de aquel impacto es un conjunto de técnicas que tienen como propósito fundamental un manejo de los asuntos humanos, de forma que sea posible un sistema de vida en armonía con la naturaleza. Esta actividad tiene que ver con las Ciencias Sociales (Economía, Sociología, Geografía, etc.) con el ámbito de las Ciencias Naturales (Geología, Biología, Química, etc.), con la gestión de empresas, etc. 
La evaluación pretende reducir al mínimo las intrusiones en el ecosistema, elevar al máximo las posibilidades de supervivencia de todas las formas de vida, reconociendo que no se sabe realmente lo que la pérdida de cualquier especie puede significar para el equilibrio biológico. El impacto no implica adversidad, ya que éste puede ser tanto negativo como positivo, la importancia del impacto dependerá de su intensidad, duración, permanencia, magnitud, y de los efectos en el ambiente (Aguilar, 2011).

Paralelamente se han ido desarrollando diferentes programas institucionales, subvenciones a proyectos de innovación, cursos de perfeccionamiento del profesorado, elaboración de materiales curriculares, etc., que tienen como principal objetivo facilitar la integración de esta nueva materia en las instituciones de educación superior. Muchos de los recursos que anteriormente se describen han sido destinados de forma prioritaria a programas de Educación Ambiental de carácter superior (Benayas \& Gutierrez).

Por tanto, se observa la necesidad de implementar la cultura ambiental como una semilla latente para la preservación del medio climático con valores y enseñanzas que se practiquen a diario con una mentalidad positiva y clara de lo que se quiere para un futuro mejor y saludable.

De acuerdo a (Polo, 2013) considera que la cultura ambiental, es la enseñanza de prevención y protección del medio ambiente, enfocado a los aspectos esenciales de la conservación de los recursos naturales (RRNN), la protección de la fauna y flora, etc., en orientación para entender las relaciones que la humanidad establece con su medio y para gestionar mejor los RRNN.

De esta forma la Organización de las Naciones Unidas para la Educación, la Ciencia y la Cultura ha insistido en que les corresponde a los institutos de educación superior pronunciarse con independencia y responsabilidad sobre los problemas éticos y sociales, y debe incorporar el ofrecer procesos formativos acorde a la situación económica y social moderna. Los temas ambientales pueden considerarse dentro de los principales problemas que enfrenta la sociedad contemporánea y que precisa de un tratamiento permanente por parte de las universidades desde la investigación y la formación profesional de pregrado y postgrado (Espinoza \& Diazgranado, 2016). 
En relación a lo anteriormente mencionado, en las conferencias internaciones sobre educación superior, se ha hecho rotunda la responsabilidad social que deben tener las universidades en cuanto a formación de liderazgo social, creación de conocimientos de alcance mundial para enfrentar retos mundiales. Entre estos se mencionan la seguridad alimentaria, el cambio climático, la gestión del agua, el diálogo intercultural, las energías renovables y la salud pública (Espinoza \& Diazgranado, 2016).

Desde el comienzo de la vida en la tierra los cambios que ha presentado la misma han sido evidentes y, es en la capa geográfica donde estos se hacen notorios, la composición de nuestro planeta ya no son lo que solían ser y su situación se complicará en el futuro; las leyes de la naturaleza y de la sociedad se asignan en su dinámica de desarrollo. (Valdés \& Rodriguez, 2013)

El medio ambiente se refiere a todo lo que rodea a los seres vivos, está conformado por elementos biofísicos (suelo, agua, clima, atmósfera, plantas, animales y microorganismos), y las relaciones con la cultura se manifiestan además de la ideología y economía. La relación que se establece entre estos elementos es lo que, desde una visión integral, conceptualiza el medio ambiente como un sistema (Arango, 2015), en la actualidad el concepto de medio ambiente está ligado al de desarrollo; esta relación nos permite entender los problemas ambientales y su vínculo con el desarrollo sustentable, el cual debe garantizar una adecuada calidad de vida para las generaciones presente y futura.

En este sentido, para (Polo, 2013) determina que el sector educativo tiene el enorme compromiso de ofrecer una educación de calidad, capaz de preparar ciudadanos competitivos y productivos, pero a la vez comprometidos con el medio ambiente y el desarrollo sustentable, es decir que cada vez requieren más en las nuevas generaciones, de una cultura ambiental adecuada y promotora del desarrollo y la riqueza sin menoscabo de las condiciones ecológicas actuales, para poder asegurarlas a las generaciones que están por venir.

El deterioro socio ambiental obliga a alterar el vínculo con la sociedad en su entorno natural, a través del desarrollo sustentable, reconociendo la presencia de limitantes en el desarrollo social, al crecimiento económico y a la explotación de los ecosistemas, a causa de la situación tecnológica que se vive en la actualidad y la posibilidad de la 
biosfera para absorber los efectos negativos de las actividades humanas. (Aguirre, 2012)

A su vez el fin de una cultural ambiental es fomentar las habilidades, destrezas, valores y conocimientos que ayuden en una ilustración de respeto al medio ambiente, y así cumplir y sostener los ejes propuestos en las estrategias nacionales medioambientales de los diferentes países del mundo. (Ministerio Medio Ambiente El Salvador, 2016)

Por otra parte (Miranda , 2013) destaca que el compromiso con valores, creencias y actitudes más próximos a una relación armónica con el medio ambiente podría convertirse en un poderoso predictor del cambio de los contextos en los comportamientos. Por ello, el análisis de la interacción entre las dimensiones mencionadas mostraría de una forma más amplia los aspectos más relevantes implicados en el desarrollo de una cultura ambiental.

Según (Rengifo \& Quitiatez) los procesos educativos que incluyen la enseñanza de la Educación Ambiental pretenden tomar conciencia del individuo y la sociedad, de igual forma la capacitación conforma un proceso que permite que los seres humanos y las sociedades desarrollen plenamente el conocimiento del entorno, interpretarlos, explicarlos y vivir sus circunstancias.

Para (Belloso, 2016) los científicos sociales han prosperado en las distintas aproximaciones conceptuales sobre el tema del comportamiento sustentable, reconociéndose la importancia de la pauta individual y colectiva en el rendimiento ambiental. Desde sus orígenes la Educación Ambiental ha ido evolucionando en dos direcciones, por una parte, a partir de un materialismo pasivo a un activismo con un marcado acento ecologista y, por otra parte, a partir del respeto por el medio ambiente como objeto exclusivo de atención hasta la toma de conciencia de su interconexión con la dimensión social, esta a su vez involucra la toma de decisiones y la autoformación de conciencia social con base en los problemas que perturban la calidad del medio ambiente.

La pedagogía tiene fuertes vínculos con la Educación Ambiental, ya que ésta tiene entre sus propósitos la formación de una conciencia sobre los problemas, ambientales y la 
puesta en práctica de acciones, para que en la sociedad se prevenga, se adapte o se contribuya a la solución de estos problemas (Flores, 2013).

Inicialmente los temas oblicuos se plantean como contenidos que hacen referencia a problemas, conflictos en la actualidad, frente a la urgencia de soluciones desde posiciones personales y colectivas, con contenidos relativos principalmente a valores y/o actitudes, a través de su programación, desarrollándose en los estudiantes, análisis y comprensión de la realidad, es decir, contextualizándolas en ámbitos en donde se expliquen los problemas del mundo actual, dotándolas de un valor funcional o de aplicación inmediata respecto a la comprensión y a la posible transformación positiva de dichos problemas así como de la realidad misma (Severiche \& Gómez, 2016).

Paralelamente se han ido desarrollando diferentes programas institucionales, subvención esos proyectos de innovación, cursos de perfeccionamiento del profesorado, elaboración de materiales curriculares, etc., que tienen como principal objetivo facilitar la integración de esta nueva materia en las instituciones de educación superior. Muchos de los recursos que anteriormente se describen han sido destinados de forma prioritaria a programas de Educación Ambiental de carácter superior (Benayas \& Gutierrez).

En el ámbito nacional, en el Ecuador es necesario que a partir de los educadores se indague el accionar de todas las ciencias, disciplinas o materias con idoneidad para educar en las relaciones que se deben establecer con el medio ambiente. La Ética, la Geografía, las Ciencias Naturales, la Biología son asignaturas que no enfatizan lo suficiente en lo referido al medio ambiental, puesto que aquellas solo se basan en teorías y no en prácticas de cuidado con el ambiente lo cual debe ser modificado por las autoridades de cada institución educativa (Sarango \& Sánchez, 2016).

El modelo de desarrollo económico actual ha traído como consecuencia una serie de problemas ambientales como la pérdida de suelos, de recursos hídricos, contaminación de residuos sólidos peligrosos y no peligrosos, contaminación visual, cambio climático, deforestación y pérdida de la biodiversidad, los cuales han afectado gravemente a los ecosistemas del planeta. De no afrontarse este deterioro a corto plazo, se pueden tener dificultades de escasez de los recursos naturales renovables y no renovables (Espejel \& Flores, 2012). 
El aprendizaje invita a reflexionar en la acción y en el proyecto en curso, cuyo objetivo esencial es el de operar un cambio en un medio (en la gente y en el medio ambiente) y cuya dinámica es participativa, implicando los diferentes actores de una situación por transformar (Severiche \& Gómez, 2016).

En el ámbito del Programa de Naciones Unidas para el Medio Ambiente (PNUMA), "La formación ambiental es comprendida, como una educación ambiental especializada, en cuanto que se dirige a un grupo restringido de profesionales" (Márquez, Casas, \& Jaula, 2017).

De la misma forma, desde su origen el planeta ha estado en permanente cambio, así lo evidencian, las distintas transformaciones en la conformación del planeta, y la evolución de las especies desde que la vida apareció en la tierra. En el mundo actual cobra cada vez más atención la actual crisis ambiental reflejada en complejos problemas como el calentamiento global, la degradación de los ecosistemas, la desaparición de especies o la contaminación (Bolaños \& Ortega, 2015).

En este contexto de acuerdo a un nuevo informe de ONU Medio Ambiente "la degradación ambiental es responsable de casi una de cada cuatro muertes en todo el mundo, lo que equivale a 12,6 millones de personas cada año y está detrás de una avalancha de problemas de salud humana y de la destrucción generalizada de ecosistemas clave" (Solheim, 2017).

\section{Conciencia ambiental}

Desde el siglo XIX es posible hablar de una conciencia ambientalista, que se caracterizó por la preocupación por el agotamiento de los recursos naturales, esenciales en los procesos productivos de una economía en plena industrialización, preocupación que a su vez dio origen a las ciencias forestales, a la ecología y a la creación de reservas naturales custodiadas por el Estado (Bolaños \& Ortega, 2015).

En este sentido la conciencia ambiental se define como "determinados factores psicológicos relacionados con la propensión de las personas a realizar comportamientos pro ambientales" (Tonello \& Valladares, 2015). 


\section{Cultura ambiental}

La cultura ambiental va siendo uno de los retos colectivos asumidos paulatinamente por la sociedad. De un lado los ciudadanos son cada vez más conscientes de la trascendencia ambiental de sus acciones en los diferentes entornos en los que se desarrolla su vida, y por otra parte las empresas van enfrentando su responsabilidad ambiental, además las administraciones están liderando iniciativas que impliquen a la población en actividades que profundicen el desarrollo sostenible.

(Severiche \& Gómez, 2016) manifiestan que esta nueva cultura ambiental, reclama la participación crítica y activa de individuos y grupos entorno a una visión convergente por y con la naturaleza. En este sentido, la cultura, como forma de vida y convivencia social de todos los pueblos, es incluyente de todas las disciplinas y, particularmente, de la educación.

Por esta razón según se considera que incrementar el nivel de cultura ambiental de la población es una prioridad, y es únicamente a través de la educación como el individuo interioriza la cultura, y es capaz de construir y producir conocimientos, reorientar sus valores, modificar sus acciones y contribuir como sujeto individual a la transformación de la realidad del medio ambiente (Miranda , 2013).

\section{Educación Ambiental}

La Educación Ambiental tiene sus orígenes en la Conferencia de las Naciones Unidas sobre el Medio Humano celebrada en junio de 1972 en Estocolmo, Suecia, donde se plantea como una alternativa en los países del mundo para promover el cuidado y conservación de la naturaleza (Espejel \& Flores, 2012).

En este sentido la Educación Ambiental es la herramienta esencial para que todos los individuos adquieran conciencia de la importancia de conservar su entorno y tengan la capacidad de modificar sus valores, conductas y costumbres, además de expandir sus conocimientos para impulsarlos a la prevención y mitigación de los problemas actuales y futuros. 
En definitiva, el reto de la Educación Ambiental, consiste en promover una nueva relación de equilibrio entre el hombre y su entorno, a fin de garantizar a las generaciones actuales y futuras un desarrollo individual y colectivo más justo, equitativo y sustentable, que pueda asegurar la conservación del soporte físico y biológico en los que se valora.

\section{MATERIALES Y MÉTODOS}

La presente investigación fue realizada en la Universidad Técnica Estatal de Quevedo (UTEQ), de orden público creada en 1984 para la colectividad y sus alrededores, brinda servicio de educación superior, cuenta con varias facultades: Facultades de Ciencias Pecuarias (FCP), Ciencias Empresariales (FCE), Ciencias Ambientales (FCAMB), Ciencias de la Ingeniería (FCI), y la Unidad de Estudios a Distancia (UED), con carreras acordes a las exigencias del medio y la demanda estudiantil.

La investigación utilizó el método analítico en la interpretación de las diferentes argumentaciones, este proceso cognoscitivo consiste en descomponer un objeto de estudio, separando cada una de las partes del todo para estudiarlas en forma individual aquella información que se obtuvo de las fuentes secundarias de libros, artículos de revistas, repositorios. De igual forma el método inductivo para dar conclusiones según los datos obtenidos, consiste en tomar conclusiones generales para obtener explicaciones particulares. Además, se recurre a la investigación de campo para obtener datos directamente de la realidad con el objeto de estudio, en la que se utilizó como instrumento la encuesta con preguntas con opciones múltiples, aplicada a estudiantes de la UTEQ, relacionadas a conocer la implementación y mantenimiento del ornato de la institución.

\section{Indicaciones metodológicas para el diagnóstico de la cultura ambiental en la Universidad Técnica Estatal de Quevedo (UTEQ).}

Se desarrolla la investigación en tres fases cada una tiene varias etapas que se desarrollan en la Figura 1, se establece el diagnóstico de la escaza cultura ambienta el al UTEQ. 


\section{Etapa 1}

Bases Preliminares

Investigación sobre la cultura ambiental en los estudiantes de la UTEQ

Utiliza el método analitico, inductivo para comprender la situación actual de la UTEQ

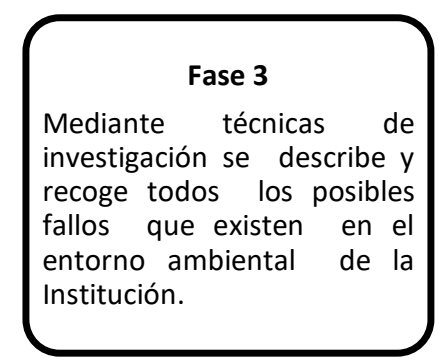

Figura 1. Etapas para el proceso.

Fuente: Elaboración propia.

Fase 1. Determinación de la cultura ambiental en los estudiantes de la UTEQ.

Objetivo: Determinar bases preliminares de la cultura ambiental al interior de la UTEQ.

Métodos y herramientas: Investigación de Campo.

Argumentación metodológica: Esta fase permite determinar las acciones cotidianas de los estudiantes en el entorno universitario, con relación al medio ambiente.

Fase 2. Identificación de actividades de la cultura ambiental universitaria.

Objetivo: Identificar la cultura ambiental estudiantil en la UTEQ.

Métodos y herramientas: Investigación descriptiva

\section{Etapa 3}

Diagnóstico estratégico de la cultura ambiental en la UTEQ.

Fase 1

Demostrar que la cultura ambiental de los estudintes incide en el medio ambiente

Fase 2

Se establece una encuesta aleoatoria como instrumento de investigación para obtener un diagnostico correctro de la situación.

\section{Fase 3}

Los resultados son discutidos y consolidados para su efiente investigación e interpretación.
Fase 1

\section{Fase 2}

la cultura ambiental con valores morales respetando e ecosistema ambiental.
Identificación de actividades a de los actores universotarios enfocandose a las actividades institucionales .

\section{Fase 3} Identificar las actividades
positivas que realiza la UTEQ.

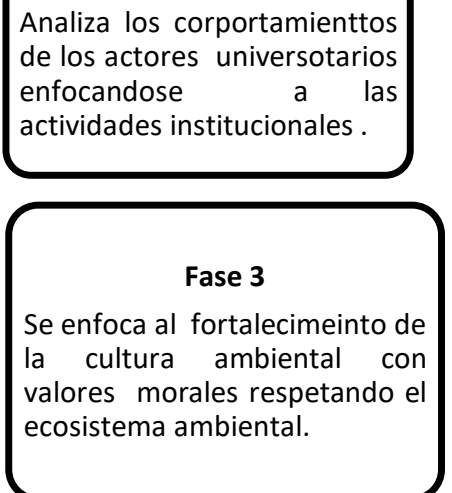


Argumentación metodológica: En esta fase selecciona las principales causas de la escaza información de cultura ambiental en la institución, mediante la descripción de hechos o sucesos que ocurre respecto al tema.

Fase 3. Determinación del diagnóstico estratégico de la cultura ambiental.

Objetivo: Determinar el nivel de la cultura ambiental de los estudiantes de la UTEQ.

Métodos y herramientas: Análisis estadístico descriptivo de resultados

Argumentación metodológica: En esta fase, aplicación del instrumento (encuesta) determinación de los niveles de conocimiento acerca de cultura ambiental.

\section{ANÁLISIS DE LOS RESULTADOS}

Enfocados en los materiales y métodos de investigación, la cultura ambiental es un compromiso de todos, examinando con eficiencia cada una de las oposiciones que se encuentran plasmados en los diferentes gráficos, conociendo las diferentes propuestas, actividades o acciones de las autoridades, docentes y estudiantes que fortalezca el cuidado medio ambiental, con objetividad sería para discutir los resultados y plantear posibles alternativas de solución. Para este fin se consultó a la comunidad universitaria respecto al cuidado del medio ambiente, los resultados se muestran en el gráfico 1.

Gráfico 1. Cuidado del medio ambiente - Comunidad Universitaria.

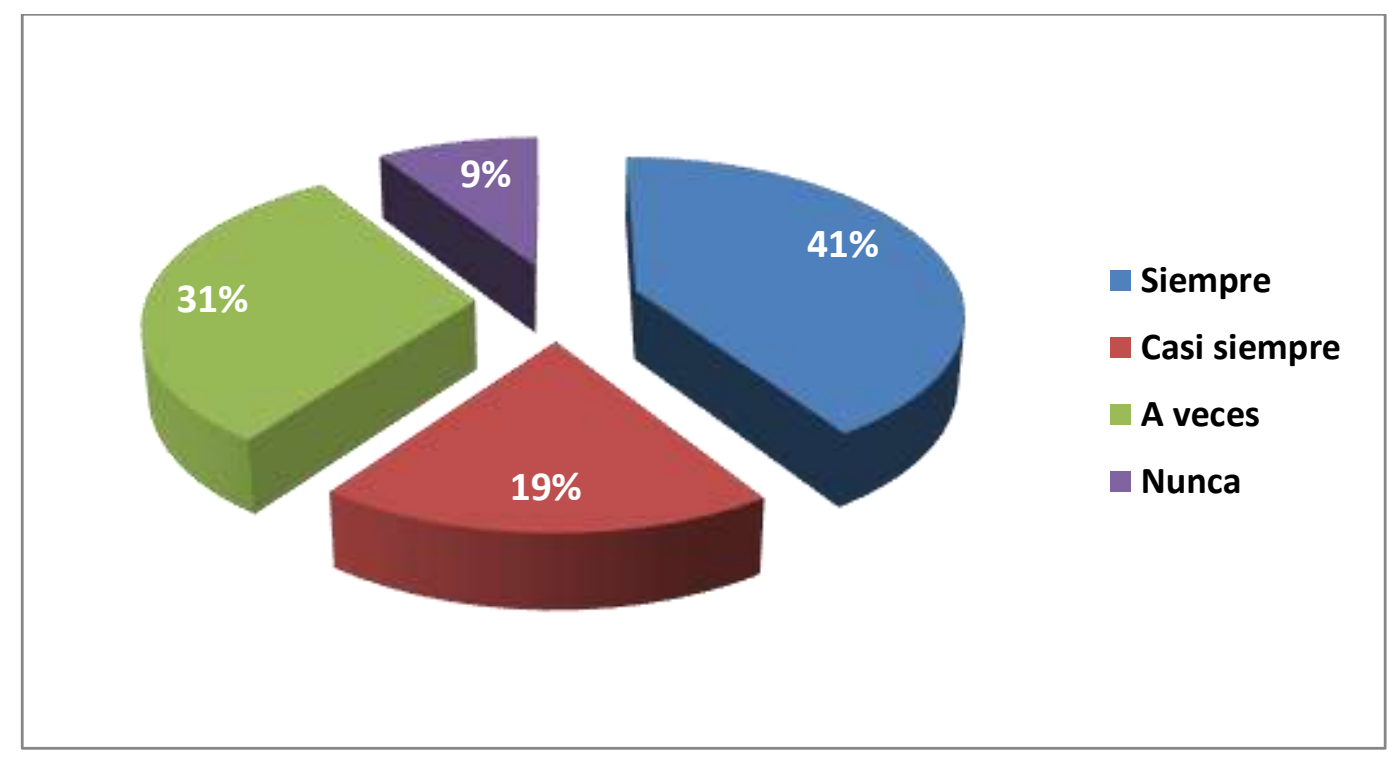

Fuente: Elaboración propia. 
La UTEQ institución educativa universitaria ubicada en el cantón Quevedo, enfatiza a la correcta preservación del medio ambiente, como uno de los factores principales para el crecimiento institucional como social, donde resalta la labor ética de todos los actores universitarios donde cada uno de ellos, el 41 por ciento siempre actúan con mentalidad positiva respecto al cuidado del medio ambiente, sin embargo, un 31 por ciento de la población universitaria casi siempre lo hace, por ende se debe fortalecer la educación ambiental aún más como un pilar permanente para la sociedad que respete y sea consciente en relación al cuidado del ambiente.

Por otra parte, un 19 por ciento establece que a veces práctica valores ambientales, asumiendo que la falta de información es la causal latente para este tipo de actitudes del individuo, sin dejar atrás al 9 por ciento restante que nunca utilizó las debidas normas de higiene en favor del ecosistema brindando una imagen lamentable para su entorno y afectando el impulso desenfrenado por un mejor medio ambiente.

Se consultó a los miembros de la comunidad estudiantil de la UTEQ, respecto al cuidado del medio ambiente, teniendo los resultados resumidos en el gráfico 2.

Gráfico 2. Importancia y cuidado del medio ambiente, estudiantes de la UTEQ.

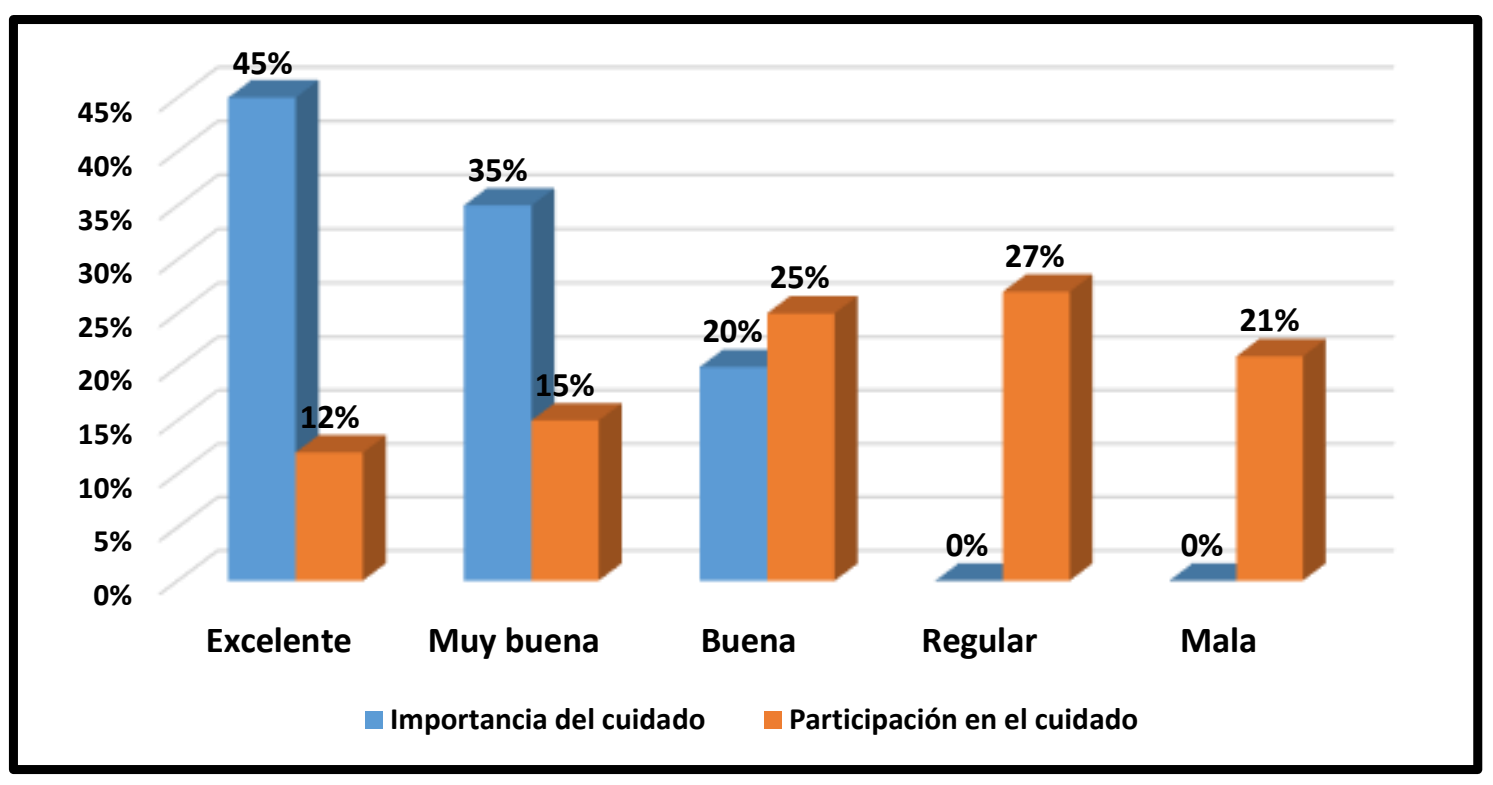

Del gráfico 2, desprende la importancia de una adecuada cultura ambiental reflejando el desempeño universitario con medidas de gestión para mantener un ecosistema saludable, la institución promueve dichas acciones favorables de tal forma que para 
los encuestados manifiestan en un 45 por ciento que su importancia es alta razón por lo que la califican de excelente, para el 35 por ciento de estos consideran que tiene una importancia de muy buena, sumando un total de 80 por ciento que en el criterio de los encuestados su importancia es alta, siendo para apenas el 20 por ciento buena por cuanto consideran, no se han generado suficientes políticas respecto al cuidado al interior de la institución.

Por otro lado al analizar la situación respecto a lo que consideran los encuestados en relación con su participación en el cuidado del medio ambiente tiene un inferencia contraria a la importancia, se observa que para el 21 por ciento de estos su participación es mala, mientras que para el 27 por ciento la consideran regular, sumando un 48 por ciento que afirman que su participación no es la adecuada al cuidado, en este sentido tanto las autoridades universitarias como los gobiernos seccionales deben establecer políticas de cuidado y protección del medio, en la misma dirección se observa en el criterio de los encuestados apenas el 25 por ciento califican de buena, para el cinco por ciento de muy buena y siendo sólo el dos por ciento de excelente, esto implica que las instituciones del cuidado del medio tienen que ejercer planes de concienciación y de comunicación respecto al cuidado del medio ambiente.

En esta misma dirección se consulta también a los estudiantes respecto al conocimiento y de métodos respecto al cuidado de la preservación del medio ambiente que conocen y que institución las está desarrollando.

Gráfico 3. Implementación de técnicas o métodos para la preservación del medio ambiente.

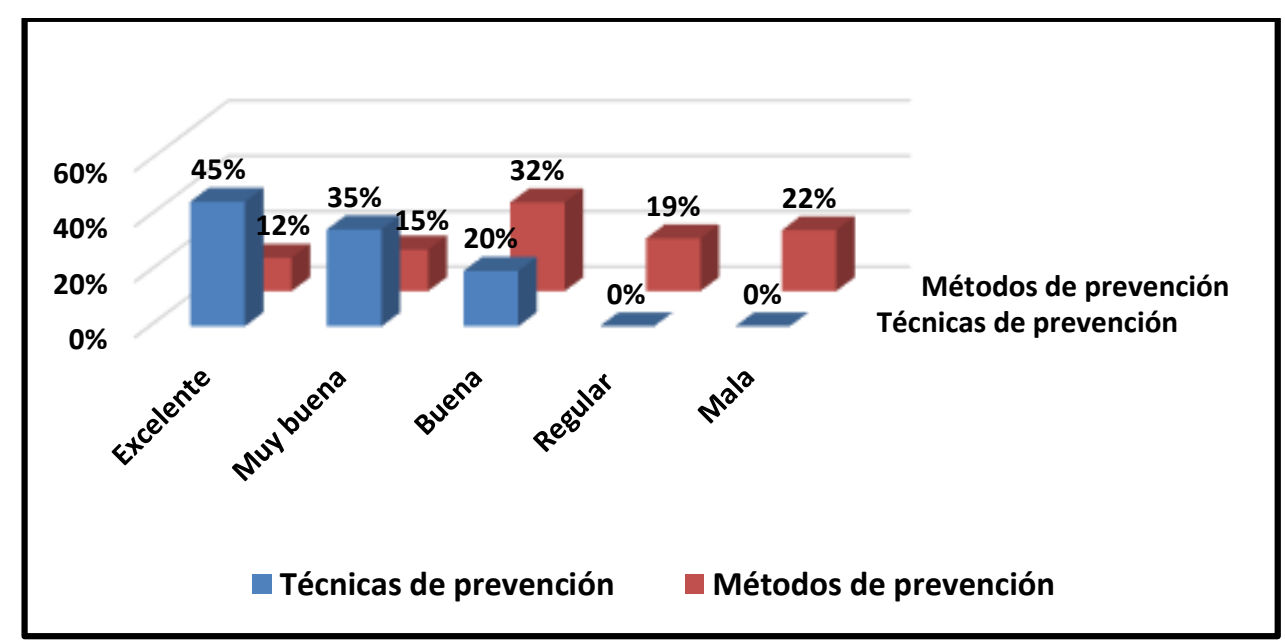

ISSN: 1390-9320, Vol. 5, No. 2, abril 2018 
Del gráfico se infiere en primera instancia respecto de las técnicas que conocen los encuestados respecto al cuidado de medio ambiente que para el 45 por ciento consideran que las charlas o programas que se han desarrollado al interior de la institución son excelentes y ellos consideran a estas como técnicas para el cuidado del mismo, mientras que para el 35 por ciento de estos consideran que estas son muy buenas refiriéndose a los medios utilizados en el interior como los tachos recicladores de basura y que constan con leyendas de clasificación de basura, por otro lado para el 20 por ciento de estos consideran que son buenas pero no efectivas ya que existe descuido por parte de la comunidad universitaria.

En este mismo sentido se observa que para el 25 por ciento de los encuestados consideran que los métodos para proteger son buenos, que falta proceso de concientización al interior de la institución, por otro lado, para apenas el 12 por ciento y el 15 por ciento de los encuestados consideran que los métodos son excelentes y muy buenos respectivamente, en esta misma dirección para el 22 por ciento consideran que los métodos son inadecuados y para el 19 por ciento lo consideran regular, manifestando que es inadecuado y que no existe involucramiento de todos en el cuidado del medio ambiente.

\section{DISCUSIÓN}

Mediante los resultados antes expuestos, refleja un amplio porcentaje de los estudiantes que practican valores éticos y morales para el cuidado del medio ambiente de manera permanente, importante para el involucramiento de estos a un proceso de mejora y de cuidado ambiental, se determina un pequeña participación tanto estudiantil como de la comunidad universitaria respecto al cuidado del medio ambiente, se denota del estudio escasos eventos al interior de la institución respecto al tema medio ambiental.

Del estudio desprende la necesidad que las instituciones educativas y gubernamentales incluyan programas de cuidado y protección al medio ambiente, además de programas de concientización ciudadana, estos aspectos son inherentes a todos en pro de propiciar la participación del conglomerado en la protección y cuidado del ambiente, en este sentido las entidades educativas tienen un rol importante para propiciar espacios de reflexión y concientizar a la población. 
El estudio determina la existencia de una escasa cultura ambiental, además de escasos programas del cuidado del medio, para las personas objeto de estudio consideran que la implementación del reciclaje es una opción. Otra opción está en el orden de programas de emprendimiento social en la recolección de desechos sólidos, además de programas estudiantiles en procesos de concientización social en temas de reciclaje, cuidado de la naturaleza y de los espacios públicos.

Finalmente, una educación también debe considerar aspectos culturales, académicos que dosifiquen al ser humano en su entorno ambiental con la participación activa de valores que forme un verdadero profesional con humildad, honestidad y sencillez, cultivando la semilla del bienestar colectivo y no individualista que margina al ecosistema natural como un simple atractivo y no como un eje transversal de desarrollo.

\section{CONCLUSIONES}

Se demostró que la UTEQ promueve a los estudiantes el uso adecuado de valores éticos y morales en beneficio del medio ambiente con actividades temporales y no permanentes.

Existen pocos eventos de concientización, del cuidado del medio ambiente, que incluya programas de desarrollo social con la participación de entidades educativas y de los gobiernos seccionales, a fin de garantizar un adecuado proceso de protección ambiental.

Se exterioriza el deseo por colaborar con la institución por parte del estudiantado, además del deseo de participar por ejemplo en programas de reciclaje, sin embargo, el estudio refleja la escasa intervención de los entes en programas de este tipo, en este sentido queda como tarea pendiente la participación de los actores universitarios y sociales.

Concomitante con esto, se determina necesario la generación de educación ambiental, en todos los escenarios sea este académico, barrial, comunitario, cantón o país en general, en este sentido la cultura ambiental es primordial para la generación de 
acciones concretas respecto a impulsar el cuidado a la naturaleza, medio ambiente y las instituciones como parte integrante del desarrollo social.

\section{REFERENCIAS BIBLIOGRÁFICAS}

Aguilar. (2011). Ecología y Medio Ambiente. Mexico: Santillana. Recuperado el 7 de Enero de 2018

Aguirre, M. (2012). Conocimientos sobre educación ambiental de los miembros de la comunidad educativa y su influenia en las prácticas ambientales que tienen los niños y niñas en los centros de desarrollo infantil públicos "Colinas del Valle" y "Goteritas". Tesis de Maestría, Universidad Central del Ecuador, Facultad de Filosofía, Letras y Ciencias de la Educación, Quito. Recuperado el 7 de Febrero de 2018, de http://www.dspace.uce.edu.ec/bitstream/25000/682/1/T-UCE-0010169.pdf

Arango. (2015). Recuperado el 1 de Enero de 2018, de http://www.banrepcultural.org/blaavirtual/ayudadetareas/ciencias/medio_ambie nte

Belloso, R. (Mayo de 2016). La educación ambiental como base cultural y estrategia para el desarrollo sostenible. Revista TELOS, 18(2), 266-281. Recuperado el 18 de Febrero de 2018, de http://www.redalyc.org/pdf/993/99345727007.pdf

Benayas, J., \& Gutierrez, J. (s.f.). Recuperado el 22 de Enero de 2018, de http://www.mapama.gob.es/es/ceneam/recursos/documentos/investigacioneducacion-ambiental-espana_tcm7-13540.pdf

Bolaños, \& Ortega. (Septiembre - Diciembre de 2015). Medio ambiente, ciencia y sociedad. Andamios, 12(29), 7-14. Recuperado el 25 de Febrero de 2018, de http://www.scielo.org.mx/scielo.php?pid=S1870-

$00632015000300007 \&$ script=sci_arttext

Espejel, A., \& Flores, A. (Octubre - Diciembre de 2012). Educación ambiental escolar y comunitaria en el nivel medio superior, Puebla-Tlaxcala, México. Revista Mexicana de Investigación Educativa, 17(55), 1173-1199. Recuperado el 28 de Febrero de 2018, de http://www.scielo.org.mx/scielo.php?script=sci_arttext\&pid=S140566662012000400008

Espinoza, \& Diazgranado. (Mayo - Agosto de 2016). La formación ambiental de los estudiantes. Recomendaciones para su consideración en la universidad. Revista 
Universidad y Sociedad, 8(3), 13 -22. Recuperado el 30 de Enero de 2018, de http://scielo.sld.cu/pdf/rus/v8n3/rus02316.pdf

Flores, C. (Enero - Junio de 2013). Diálogos entre la pedagogía y la educación ambiental. Educación y Desarrollo Social, 7(1). Recuperado el 19 de Febrero de 2018, de http://artemisa.unicauca.edu.co/ gerardorengifo/Documentos/2014_InvCien_cl ase_asesoria\%20Salida\%20PNN_educacion_Amb.pdf

Márquez, D., Casas, M., \& Jaula, J. (2017). La formación ambiental en la universidad cubana. Revista Universidad y Sociedad, 9(3), 207-213. Obtenido de http://scielo.sld.cu/scielo.php?script=sci_arttext\&pid=S221836202017000300032

Ministerio Medio Ambiente El Salvador. (7 de Abril de 2016). Recuperado el 12 de Febrero de 2018, de http://www.marn.gob.sv/educacion-y-cultura-ambiental-2/ Miranda . (Julio - Diciembre de 2013). Revista Producción + Limpia, 8(2), 94-105. Recuperado el 16 de Febrero de 2018, de http://repository.lasallista.edu.co:8080/ojs/index.php/pl/article/view/527/276

Miranda. (2013). Cultura ambiental: un estudio desde las dimensiones de valor, creencias, actitudes y comportamientos ambientale. Producción + Limpia, 8(2). Recuperado el 5 de Enero de 2018, de http://www.scielo.org.co/scielo.php?pid=S1909-

04552013000200010\&script=sci_abstract\&tIng=es

Polo, J. (Octubre - Diciembre de 2013). El Estado y la educación Ambiental Comunitaria en el Perú. Revista Acta Médica Peruana, 30(4), 141-147. Recuperado el 25 de Enero de 2018, de http://www.redalyc.org/pdf/966/96629462017.pdf

Rengifo, \& Quitiatez. (s.f.). Recuperado el 17 de Febrero de 2018, de La Educacion Ambiental una estrategia pedagógica que contribuye a la solución de la problemática ambiental en Colombia: http://www.ub.edu/geocrit/coloquio2012/actas/06-B-Rengifo.pdf

Sarango, J., \& Sánchez, S. (2016). Educación ambiental. ¿Por qué la Historia? Revista Universidad y Sociedad, 8(3), 184 -187. Recuperado el 22 de Febrero de 2018, de http://scielo.sld.cu/pdf/rus/v8n3/rus25316.pdf

Severiche, \& Gómez. (Mayo - Agosto de 2016). La educación ambiental como base cultural y estrategia para el desarrollo sostenible. Telos, 18(2), 266-281. Recuperado el 26 de Febrero de 2018, de http://www.redalyc.org/pdf/993/99345727007.pdf 
Solheim, E. (16 de Noviembre de 2017). Hacia un planeta sin contaminación. Naciones Unidas , Nairobi. Recuperado el 24 de Febrero de 2018, de https://papersmart.unon.org/resolution/uploads/k1708350s.pdf

Tonello, G., \& Valladares, N. (Marzo de 2015). Conciencia ambiental y conducta sustentable relacionada con el uso de energía para iluminación. Gestión y Ambiente, 18(1). Recuperado el 25 de Febrero de 2018, de http://ri.conicet.gov.ar/bitstream/handle/11336/10657/CONICET_Digital_Nro.14 229. pdf? sequence $=1$ \&isAllowed $=y$

Valdés , \& Rodriguez. (2013). La educación Ambiental y Desarrollo Sostenible: estrategias de integración interdisciplinarias curricular e institucional en los programas, proyectos y buenas parcticas en las univeridades, escuelas, familias y comunidades en Cuba. Educación Cubana. Recuperado el 3 de Febrero de 2018,

de http://www.unesco.org/new/fileadmin/MULTIMEDIA/FIELD/Havana/pdf/Curso_1 5.pdf 